\title{
Epidemiology, risk factors and prognosis of cardiovascular disease in the Coronavirus Disease 2019 (COVID-19) pandemic era: a systematic review
}

\author{
Garyfallia Pepera ${ }^{1}$, Marina-Sofia Tribali ${ }^{1}$, Ladislav Batalik ${ }^{2,3, *}$, Ivo Petrov ${ }^{4}$, \\ Jannis Papathanasiou ${ }^{5,6}$
}

\author{
${ }^{1}$ Physiotherapy Department, Faculty of Health Sciences, University of Thessaly, 35100 Lamia, Greece \\ ${ }^{2}$ Department of Rehabilitation, University Hospital Brno, 62500 Brno, Czech Republic \\ ${ }^{3}$ Department of Public Health, Faculty of Medicine, Masaryk University Brno, 60177 Brno, Czech Republic \\ ${ }^{4}$ Clinic of Cardiology and Angiology, Acibadem City Clinic Cardiovascular Center, 1407 Sofia, Bulgaria \\ ${ }^{5}$ Department of Medical Imaging, Allergology \& Physiotherapy, Faculty of Dental Medicine, Medical University of Plovdiv, 4002 Plovdiv, Bulgaria \\ ${ }^{6}$ Department of Kinesitherapy, Faculty of Public Health "Prof. Dr. Tzecomir Vodenicharov, Ph.D”, Medical University of Sofia, 1431 Sofia, Bulgaria \\ *Correspondence: batalik.ladislav@fnbrno.cz (Ladislav Batalik) \\ Academic Editor: Peter A. McCullough
}

Submitted: 26 September 2021 Revised: 24 November 2021 Accepted: 6 December 2021 Published: 17 January 2022

\begin{abstract}
Background: Since the emergence of severe acute respiratory syndrome coronavirus 2 (SARS-CoV-2) from China, the novel coronavirus disease 2019 (COVID-19) has caused more than five milion deaths worldwide. Several studies have elucidated the role of risk factors in the prognosis of cardiovascular disease (CVD) in the progression of COVID-19 pandemic. This systematic review assesses the link between COVID-19 and cardiovascular risk factors, and investigates the prognosis in the case of myocardial injury. Methods: A literature search was performed to identify relevant articles in Pubmed, MEDLINE, Elsevier, and Google Scholar the last two years using the terms: COVID-19, CVD, risk factors, cardiovascular risk factors, SARS-CoV-2, lockdown, hypertension, and diabetes mellitus. Exclusion criteria were the studies associated with pediatric and pregnant COVID-19 patients. Results: After screening through 3071 articles, 10 studies were included in this review that captured the findings from 3912 participants. Included studies found that preexisting CVD was linked to worse outcomes and increased risk of death in patients with COVID-19, whereas COVID-19 itself also induced myocardial injury, arrhythmia, acute coronary syndrome, and venous thromboembolism. Conclusions: Cardiovascular risk factors such as hypertension, diabetes mellitus, and obesity were associated with intensive care unit admission and poor prognosis. Cardiovascular risk factors are crucial for the progression of COVID-19, and infected patients should be constantly monitored and follow strict hygiene and decrease their social interactions.
\end{abstract}

Keywords: Cardiovascular disease; COVID-19; SARS-CoV-2; Coronavirus disease pandemic; Risk factors; Epidemiology; Cardiovascular risk factors; Prognosis; Systematic review

\section{Introduction}

Coronavirus disease (COVID-19) is a new and significant threat that has increased mortality worldwide. COVID-19 is a severe illness caused by severe acute respiratory syndrome coronavirus 2 (SARS-CoV-2) that originated from Wuhan, China at the end of 2019 [1]. According to the World Health Organization (WHO) (2021), SARSCoV-2 is a highly transmissible and pathogenic coronavirus, which induces a severe acute respiratory syndrome, that has caused more than 2 million deaths in the first 12 months of the pandemic, in addition to significant economic and social disturbance around the world [2]. The SARSCoV-2 infection continues to spread with more than 200 million confirmed cases, and 223 affected countries, areas, or territories [2].

In February 2020, within a matter of 2 months since the beginning of the outbreak of COVID-19, more than 82 000 confirmed cases and 2800 deaths were reported, and the number of infections has risen daily since then $[1,3]$. Clinical features of COVID-19 may fluctuate from asymptomatic or mild respiratory symptoms to severe lifethreatening bilateral pneumonia, and respiratory and cardiac failure [4]. Approximately 14\% of COVID-19 cases were severe that required hospitalization, and 5\% were critical cases characterized with manifested airway failure, septic shock, and/or multi-organic dysfunction or failure - requiring intensive care [5]. The chest computed tomography (CT) shows that COVID-19 manifests as pure groundglass opacities, consolidation, and "air bronchogram sign" [6]. The period between infection and the appearance of symptoms varies between individuals, but the period can be usually 14 days long. Troponin T elevation, cardiac dysfunction, and arrhythmias are complications in hospitalized COVID19 patients and are associated with adverse outcomes [7,8]. Assessment of the early development of persistent myocardial injury is a useful prognostic tool for severe COVID-19 
[9]. Systemic immune over activation due to SARS-CoV-2 infection triggers a cytokine storm, which is noteworthy in critically ill patients with COVID-19 and the evidence from current studies has displayed that the cytokine storm may be a substantial factor in disease progression, even resulting in several organ failures and death [10,11].

As mentioned above, COVID-19 generally infects people who are immunocompromised, and older adults with underlying diseases are more vulnerable. Clinical manifestations differ across age groups. Older men ( $>60$ years old) with comorbidities are more likely to develop a severe respiratory disease that requires hospitalization, increasing the mortality risk, whereas most young people and children have only mild diseases (non-pneumonia or mild pneumonia) or are asymptomatic $[12,13]$. However, a small study in Wuhan enclosed 85 fatal cases of COVID-19, in which most cases were males aged over 50 years with noncommunicable chronic diseases [14]. Healthcare workers are a high-risk group, and they represent $3.8 \%$ of infected individuals in China [15]. Data is constantly being updated, but the caveat is that everyone is susceptible to acquiring COVID-19.

Correspondingly, cardiovascular disease (CVD) is the leading cause of mortality and morbidity worldwide. Management of CVD and its risk factors may prevent future CVD incidents such as myocardial infarction, stroke, and death from CVD [16]. There have been approximately 17.9 million people global deaths from CVD in 2015. Ischemic heart disease (IHD) and stroke were the top two leading reasons for CVD health loss worldwide. By 2030 more than 22.2 million individuals will die annually from CVD $[17,18]$.

The pandemic has led to extensive social change in many countries. Governments have enforced restrictions on citizen movement, work from home, distance learning, and social interactions. Particularly groups such as cardiology, respiratory, diabetic patients and older people should limit their social interactions because of their increased vulnerability to infection. This systematic review assesses the link between COVID-19 and CVD risk factors, and investigates the prognosis in the case of myocardial injury.

\section{Methods}

\subsection{Search strategy}

This systematic review followed the PRISMA guidelines (CRD42021264985, PROSPERO). A comprehensive literature search was performed on Pubmed, MEDLINE, Elsevier, and Google Scholar from 1st December 2019, before the first confirmed case of COVID-19, to 14th April 2021. The search strategy consisted of different combinations of the following search terms: "COVID- 19", SARSCoV-2", "cardiovascular disease", "risk factors", "cardiovascular risk factors", "hypertension", "diabetes mellitus".

\subsection{Study and cohort selection}

Article screening was performed by two independent reviewers, and consensus was resolved by a senior author. Studies reporting the incidence of stroke in patients with COVID-19 were identified separately. The full texts of any potentially eligible articles were screened against the inclusion and exclusion criteria. All English-language studies (case reports, case series, and observational cohort studies) that reported CVD in COVID-19 patients were included. Systematic reviews, meta-analyses, retrospective studies, observational studies, and clinical guidelines regarding the cardiovascular implications, epidemiology, prognosis, and risk factors related to COVID-19 were included. The reference lists of these articles were additionally screened to identify relevant studies not found from the database search. Articles with pediatric incidents and pregnant patients were excluded. Narrative reviews, duplicate studies, editorial or opinion articles, grey literature and conference papers were excluded. Studies were classified with an evidence rating (I to IV) [19].

\section{Results}

\subsection{Included studies}

The search strategy identified a total of 3071 articles, from which 2271 articles were excluded after initial screening. Further screening was performed on the remaining 800 articles, and 569 were excluded because they were not appropriate for the aims of this systematic review. In the fulltext evaluation , 231 articles were additionally excluded because they did not fit the scope of this systematic review. In total, 10 articles met the selection criteria and were included in the systematic review. The reviewers had $99 \%$ agreement in the selection of eligible articles. Fig. 1 illustrates the study search strategy and selection process. Six studies were published in China, three in the United States, and one in Japan.

\subsection{Cardiovascular risk factors}

Cardiovascular risk factors are mainly divided into two categories: modifiable and nonmodifiable. The main modifiable cardiovascular risk factors are dyslipidemia (i.e., exceptionally high levels of low-density lipoprotein (LDL) and low levels of high-density lipoprotein (HDL)), hypertension, diabetes mellitus, obesity, smoking history, alcohol consumption, physical inactivity, inflammatory markers, hyperhomo-cysteinemia and psychological factors. The nonmodifiable risk factors are age, sex, and hereditary factors [20,21]. Greece has among the highest number of deaths in Europe from ischemic heart disease and stroke primarily because of challenges with maintaining high patient adherence to medical consultations and treatment [22].

The primary modifiable cardiovascular risk factors such as hypertension, diabetes mellitus, obesity, smoking, inflammatory markers — likely intersect with risk factors 


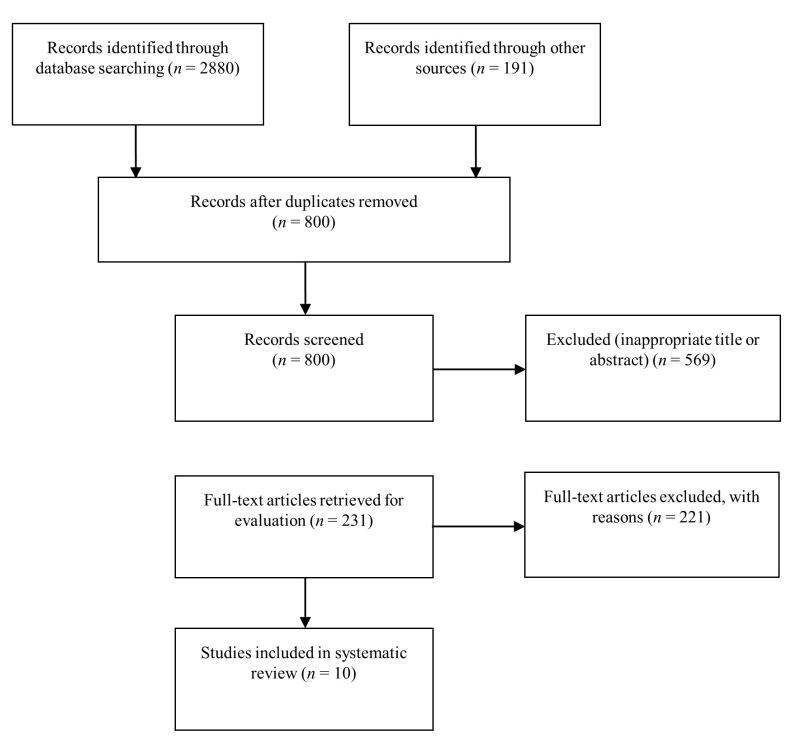

Fig. 1. Flow diagram of the study selection process.

of the severe COVID-19, as presented below. In addition, older age is a significant risk factor for fatal outcomes.

\subsection{Hypertension \& diabetes mellitus}

Hypertension and diabetes are probably the most prevalent comorbidities among individuals with COVID19, with hypertension being the most common comorbidity. However, early studies on COVID-19 show mixed results concerning hypertension and diabetes mellitus.

In many cases, diabetes mellitus and hypertension require hospitalization and possible ICU admission, and prevalence increase with age, especially in older patients [6,10,23-26]. Infection susceptibility for adult patients with diabetes mellitus is described extensively by the literature comprising of aggravated inflammatory storm, immunodeficiency, lung injury, increased infectivity, and virulence of SARS-CoV-2 in diabetes mellitus [25]. As for mechanisms of COVID-19, infection may cause the destruction of pancreatic beta cells, decreased pancreatic insulin content, and changes in the host's ability to respond generally to glucose tolerance tests $[15,27]$. Inefficient control of glycemic levels may be crucial for adult patients with diabetes mellitus infected by SARS-CoV-2 in various ways. For instance, angiotensin-converting enzyme inhibitors (ACEi) withdrawal, corticosteroid therapy, septic status derived from high glycose levels, and absence of patient monitoring with diabetes mellitus may lead to poor prognosis [28].

A recent meta-analysis of Silverio et al. [28] that involved 45 studies encompassing 18300 patients investigated the effect of cardiovascular risk factors on inhospital COVID-19 mortality. This meta-analysis found that patients with any type of diabetes who were infected by SARS-CoV-2, compared to those without diabetes mellitus, had a higher risk for in-hospital mortality. This rela- tionship was independent from age. Furthermore, a recent retrospective cohort study demonstrated that patients with diabetes who were on insulin therapy might have a higher risk of disease progression and poor prognosis [29].

When compared with non-severe patients, those with severe illness or admission to the ICU were more likely to have one or more comorbidities $(p<0.001)$, including chronic obstructive pulmonary disease $(p<0.001)$, diabetes mellitus ( $p<0.001)$, hypertension $(p<0.001)$, coronary heart disease $(p<0.001)$, cerebrovascular diseases $(p$ $<0.05)$, and cancer $(p<0.05)$ [30,31]. However, no significant differences were observed for patients with chronic liver disease $(p=0.19)$ and chronic renal disease $(p=0.40)$ [32].

The meta-analysis by de Almeida-Pititto et al. [33] reported a moderate positive relationship between hypertension and diabetes mellitus with COVID-19 mortality and severity. Furthermore, age was associated with a higher risk of in-patient hospital mortality in patients with COVID-19. Therefore, the need for unique strategies to protect vulnerable groups such as the elderly is proving vital. Interestingly, assessing cardiovascular risk, comorbidities through Charlson comorbidity index, socioeconomic status, and echocardiographic measures, can predict the severity and mortality after SARS-CoV-2 infection [34]. On the contrary, other studies reported that male sex, hypertension, and smoking did not independently correlate with fatal outcomes [29].

\subsection{Obesity}

Comparable to diabetes mellitus, the global burden of obesity is also very high. Obesity is a critical risk factor for complications and mortality from various infections [35]. Patients with BMI $\geq 35 \mathrm{~kg} / \mathrm{m}^{2}$, specifically those with heart disease, are at a high risk for severe COVID-19 [36]. The literature included in this study indicated that a poor prognosis accompanied with obesity for the SARS-CoV-2 virus that involved severe COVID-19, ICU care, invasive mechanical ventilation (IMV) use, and CVD progress, particularly amongst younger patients. However, the assocation between obesity and hospital mortality is unclear [36-39].

\subsection{Smoking}

The included literature reported mixed findings pertaining to the progression in COVID - 19 from smoking. For instance, active smoking was not related to an increased risk of severe COVID-19 in a meta-analysis by Lippi \& Henry [40]. Additionally, an observational study claimed countries with a high prevalence of smokers also had comparatively lower COVID-19 mortality [41]. On the contrary, a recent meta-analyses found that smoking history contributed to the development of COVID-19 and provoked susceptibility to COVID-19 infection. Non-smokers showed better cardiorespiratory fitness and prognosis in the case of illness than smokers, avoiding in-hospital outcomes [32,42-44]. 


\subsection{Inflammation}

Elevated levels of cytokines - such as C-reactive protein (CRP), tumor necrosis factor (TNF)- $\alpha$, interleukin-2 re-ceptor (IL-2R), IL-1 $\beta$, IL-6, IL-8 - have been observed in hospitalized COVID-19 patients [45]. Studies included in this review considered that CRP, TNF- $\alpha$, and IL-6 were risk factors that independently predicted cardiac injury in severe and critically ill patients with COVID-19 [6,10]. An increased number of cytokines can provoke myocardial injury and septic shock, multiorgan failure, and circulatory failure in some patients [46]. Given that other medical conditions such as heart failure lead a plethora of cytokines, the virus may additionall aggravate existing disease.

Inflammation is central to immunse response to tissue damage [47]. Inflammation helps to remove injurious stimuli like infections and trauma, and initiate tissue regeneration. The whole immune mechanism is inflammo-centric, with adaptive immunity being employed to enhance the effectiveness of this core response through debris clearance and tissue reconstruction. Inflammation is initially protective [47]. Yet, nutrition excess can disturb the tissue healing process because the nutrition from virus degradation and the damaged tissue with the excessive nutrition already in the body will be transform into lipid intermediates and be deposited in healthy non-adipose tissues, causing lipotoxicity and further tissue damage. Thus, over-nutrition may lead to a vicious cycle of excessive lean mass (like protein) broken down and lipid intermediates piling up, fuel excessive inflammation and lead to a cytokine storm in the severe cases of COVID-19.

Moreover, it has been identified that the COVID-19 infection usually leads to a condition known as "silent hypoxemia". The patient, despite the severe hypoxemia individually, does not feel dyspneic [48]. The prevalence and outcomes of cases with silent hypoxemia, which dominates the initial stage of infection, are caused by more vascular involvement than a lung injury $[49,50]$. However, the pathophysiological mechanism remains a matter of discussion.

\subsection{Dyslipidemia}

Another intriguing issue is the relationship between dyslipidemia and COVID-19. According to the current literature, cytokine storms may complicate dyslipidemia, reduce HDL and LDL levels, and increase triglycerides and lipoprotein oxidation [51]. Dyslipidemia is a prognostic factor for severity and mortality in patients with COVID19 [52]. Moreover, the use of statins indicate the presence of underlying comorbidities, exposing increased risk of severe COVID-19 [53]. Additionally, the evidence found in this review showed hypertension to the most common symptom to cause severe COVID-19 (59.3\%), closely followed by obesity (48.7\%), chronic lung disease (19.8\%), metabolic disease (43.6\%), and CVD (35.6\%) (Fig. 2, Ref. [54]). However, studies pertaining to pediatric populations and pregnant patients were excluded from this review. Ta- ble 1 (Ref. [10,12,27,29-31,34,39,45,55] summarizes the results of the above studies. Table 2 (Ref. [10,12,27,29$31,34,39,45,55]$ refers to the levels of evidence of included studies. The majority of the studies were level II or III in evidence quality (88\%) [19].

\section{Discussion}

This systematic review assessed the link between COVID-19 and CVD risk factors, and investigated prognosis in the case of myocardial injury. The review showed that preexisting CVD is associated with worse outcomes and increased risk of mortality in patients with COVID-19, whereas COVID-19 itself may also induce myocardial injury, acute myocardial infarction, blood thrombosis or arrhythmias. Moreover, cardiovascular risk factors are crucial for understanding the progression of COVID-19. Infected patients should be constantly monitored, follow strict hygiene protocols, and decrease their social interactions.

\subsection{Prognosis of COVID-19 in patients with cardiovascular risk factors or CVD-myocardial injury}

$\mathrm{CV}$ risk factors, preexisting CVD, and myocardial injury are negative prognostic predictors of severe cases of COVID-19 based on our current understanding of SARSCoV-2 [56]. Common pre-existing diseases may be risk factors for a poor prognosis, including CVD (10.5\%), hypertension $(6 \%)$, diabetes mellitus $(7.3 \%)$, chronic respiratory disease $(6.3 \%)$, and cancer (5.6\%) [15]. Similarly, smoking history and older age may be high-risk factors. Cardiovascular implications may worsen prognosis in patients with COVID-19, emphasizing the importance of advanced detection and optimal therapeutic strategies. Myocardial injury is a prevalent complication in hospitalized COVID-19 patients, with or without previous CVD, that may present a significant association with in-hospital mortality and a poor prognosis [23].

Yang et al. [39], in their study of 224 patients, suggested that the SARS-CoV-2 virus can induce myocardial injury, increase clinical progression, and worsen prognosis. Compared with survivors, non-survivors were older, with severe inflammation, more comorbidities, higher levels of myocardial biomarkers, and lower oxygen saturation. However, as the myocardial cells are not first cells - like the respiratory epithelial cells or the gastrointestinal epithelial cells which have direct contact with the virus particles - it is unlikely that SARS-CoV-2 viral infection has a direct impact on myocardial injury. It is more likely that viral infection starts by inducing the deaths of respiratory epithelial cells through apoptosis, necrosis, or necroptosis. Cell death in the respiratory tract and localized inflammation is an effective immune response to SARS-CoV-2 viral infection that ultimately contains the virus from spreading to other organs of the body [57-59]. Yet the immunological degradation of cells damaged by virus particles may create a transient surge of nutrition supply to the whole body, which 


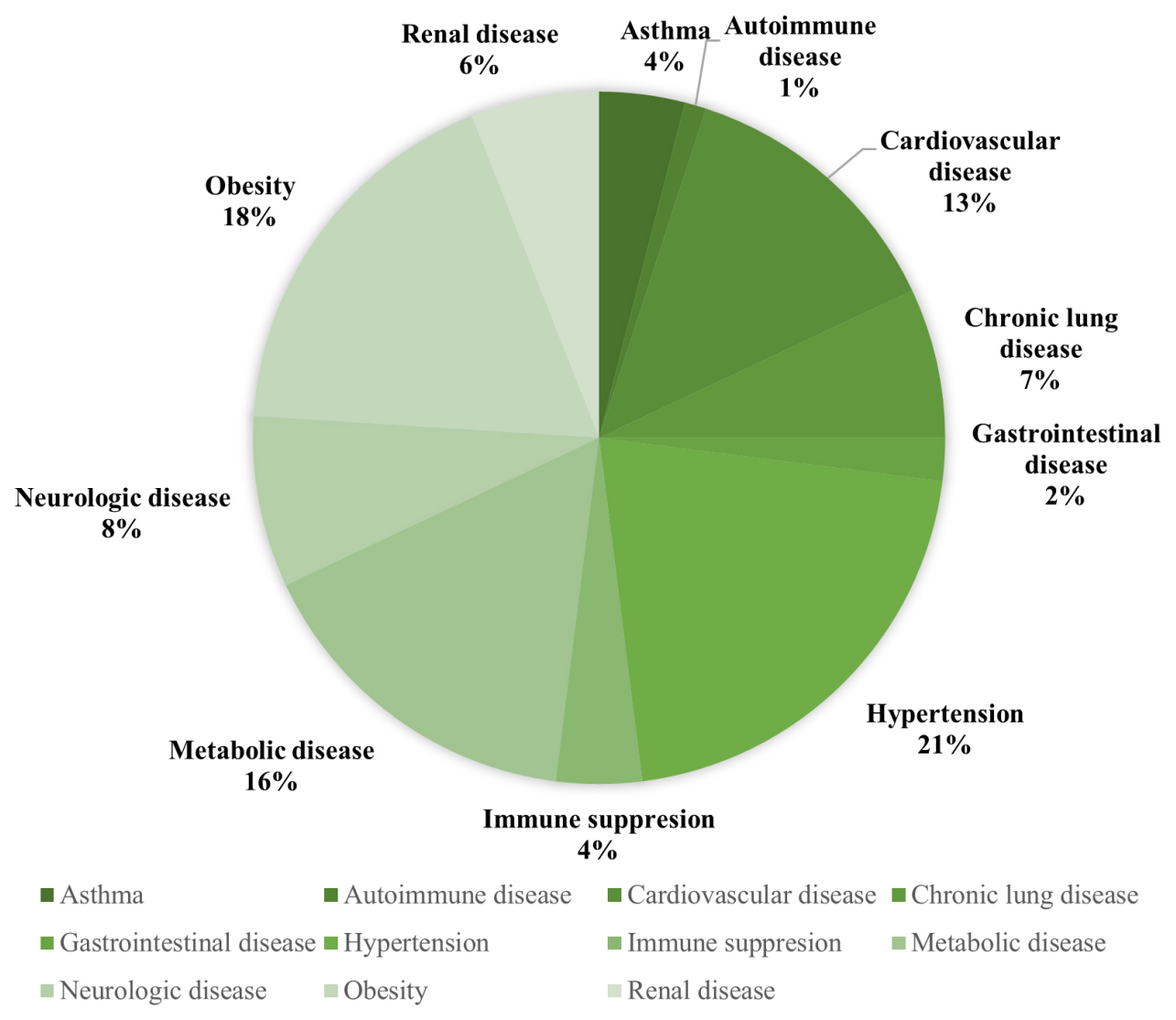

Fig. 2. Underlying comorbidities in COVID-19 patients [54]. Hospitalization data were restricted to cases between March 2020 and July 2021. Throughout this time, data was collected from hospitalized adults aged $\geq 18$ years.

may create an overnutrition state, possibly leading to lipotoxicity in the whole body in some patients [60], that may also lead to damaged myocardial cells [61-65]. Basically, CVD is more likely to be caused by the indirect affect of the virus infection on immune system degradation and liptoxicity.

Another interesting point is the effect of SARS-CoV2 on the cardiovascular system, as it may cause or conduce to acute cardiovascular events, microvascular thrombosis, myocardial damage, and arrhythmias [12,66-68].

Summarizing, the evidence from the studies suggested that prior CVD, older age, male sex, the early requirement for oxygen supplementation, higher respiratory rates, modified mental state, and laboratory abnormalities, cytokine storm, and higher troponin $\mathrm{T}$ concentrations were correlated with adverse prognosis [23,55]. The findings of this review show that the myocardial injury is an ordinary clinical manifestation of COVID-19 and a significant risk factor for poor prognosis.

\subsection{Cardiovascular rehabilitation and exercise in post-COVID-19 era}

Lai et al. [69] claimed that the clinical impacts of from SARS-CoV-2 pneumonia is correlated to a high CVD risk. Generally, the findings of included literature emphasize the importance of special preventive and protective strategies and tailored therapeutic approaches. First, cardiovascular patients and patients with several cardiovascular risk factors should be monitored consistently for microvascular, thrombosis, arrhythmias, and acute cardiovascular events. After hospitalization, an interdisciplinary approach is crucial for patient management [70]. Especially for cardiovascular patients, exercise-based cardiopulmonary rehabilitation may be a cornerstone for managing the side effects of SARS$\mathrm{CoV}-2$, starting with in-hospital early mobilization.

A consensus statement by Davies et al. [71] provided valuable recommendations for rehabilitation in postCOVID-19 era. However, there is a lack of evidence about the effect of rehabilitation based on exercise training in COVID-19 survivors. A recent review described an exercise-based rehabilitation program, physiotherapy airway clearance techniques, and techniques focused on ventilation and dyspnea management from existed literature and studies in non-COVID-19 patients [71]. However, it lays the foundations for future research to develop exercisebased rehabilitation and guidelines in COVID-19 survivors [72-74]. In any case, exercise is a valuable therapeutic approach with various benefits for respiratory, cardiovascular, and musculoskeletal systems, and future guidelines will be important tools for healthcare workers [75-78]. 
Table 1. Impact of cardiovascular risk factors in COVID-19.

\begin{tabular}{|c|c|c|c|}
\hline Study & Number of patients (characteristics) & Age & Results \\
\hline \multirow{5}{*}{ Huang et al. (2020) [10] } & $n=41$ & \multirow{5}{*}{ Median age: 49} & Admission in ICU:13/41, 8/41: diabetes \\
\hline & & & mellitus, 6/41: hypertension \\
\hline & Non-survivors: 6 & & 6/41: cardiovascular disease 12/41: \\
\hline & & & developed ARDS \\
\hline & & & $\begin{array}{l}\text { 5/41: acute cardiac injury ICU patients, } \\
\text { increased inflammatory markers }\end{array}$ \\
\hline \multirow{3}{*}{ Guo et al. (2020) [27] } & $n=174$ & \multirow{3}{*}{ Median age: 59} & Diabetes mellitus is a severe risk factor for \\
\hline & Patients with Diabetes mellitus: 24 & & organ damage, elevated inflammatory factors \\
\hline & & & or hypercoagulability \\
\hline \multirow{2}{*}{ Ghany et al. (2020) [34] } & $n=400$ & \multirow{2}{*}{$72 \pm 8$} & Age, socioeconomic status, Charlson score \\
\hline & Hospitalized: 244 & & $(2.9 \pm 1.4)$, systolic \\
\hline \multirow{4}{*}{ Shang et al. (2021) [29] } & $n=584$ & \multirow{4}{*}{ Median age: 59} & Blood pressure, body mass index, grade 2 \\
\hline & & & or 3 diastolic dysfunction \\
\hline & Non-survivors: 40 & & moderate or severe left ventricular \\
\hline & & & hypertrophy \\
\hline \multirow{2}{*}{ Yang et al. (2021) [39] } & $n=203$ & \multirow{2}{*}{$>80$} & A significant predictor of hospitalization \\
\hline & Non survivors: 58 & & and ARDS \\
\hline \multirow{2}{*}{ Pareek et al. (2021) [55] } & $n=586$ & \multirow{2}{*}{ Median age: 58} & Diabetes mellitus is an independent risk factor \\
\hline & Non-survivors: 82 & & for the prognosis of COVID-19 \\
\hline Xia et al. (2021) [45] & $n=330$ & Median age: 67 & $17 / 40$ non-survivors had diabetes mellitus \\
\hline \multirow{2}{*}{ Guan et al. (2021) [12] } & $n=463$ & \multirow{2}{*}{ Median age: 61} & \multirow{2}{*}{ non-survivors: older with comorbidities } \\
\hline & Non-survivors: 60 & & \\
\hline \multirow{2}{*}{ Kuroda et al. (2021) [31] } & $n=693$ & \multirow{2}{*}{$68 \pm 15$} & $61 \%$ cases with at least one risk factor, \\
\hline & Non-survivors: 108 & & diabetes mellitus most prevalent \\
\hline \multirow{2}{*}{ Hsu et al. (2021) [30] } & $n=438$ & \multirow{2}{*}{$65 \pm 13$} & High prevalence of diabetes mellitus and \\
\hline & Non-survivors: 109 & & ischemic heart disease \\
\hline
\end{tabular}

ARDS, Acute Respiratory Distress Syndrome; ICU, Intensive Care Unit; CRP, C-reactive protein; TNF- $\alpha$, Tumor Necrosis Factor- $\alpha$.

Table 2. Levels of evidence of included studies.

\begin{tabular}{lc}
\hline Studies & Level of evidence \\
\hline Huang et al. (2020) [10] & II \\
Guo et al. (2020) [27] & III \\
Shang et al. (2021) [29] & III \\
Yang et al. (2021) [39] & I \\
Pareek et al. (2021) [55] & II \\
Xia et al. (2021) [45] & III \\
Guan et al. $(2021)[12]$ & III \\
Ghany et al. (2020) [34] & II \\
Kuroda et al. (2021) [31] & II \\
Hsu et al. (2021) [30] & II \\
\hline
\end{tabular}

\section{Conclusions}

CVD and cardiovascular risk factors play a pivotal role in the development of COVID-19, leading to a higher risk of ICU hospitalization and mortality. Correspondingly, SARS-CoV-2 can induce myocardial injury and cardiovascular side effects. Hypertension is a leading cause of poor progression among patients with COVID-19, followed by diabetes mellitus, CVD, smoking, inflammatory markers storm, obesity, and older age. The findings of this review emphasize how preventive and tailored management of infected patients with comorbidities is essential for survivorship and the prevention of side effects. These findings will essential for a better understanding of COVID-19 and the cardiovascular system (indications for risk estimation, diagnosis, and treatment selection), and future investigations regarding exercise-based rehabilitation for COVID-19 survivors.

\section{Abbreviations}

COVID-19, novel coronavirus disease 2019; SARS$\mathrm{CoV}-2$, severe acute respiratory syndrome coronavirus 2; SARS, severe acute respiratory syndrome; ICU, intensive care unit; CT, computed tomography; CVD, cardiovascular disease; IHD, ischemic heart disease; ACE2, angiotensinconverting enzyme-2; LDL, low-density lipoprotein; HDL, high-density lipoprotein; ACEi, angiotensin-converting enzyme inhibitors; CRP, C-reactive protein; TNF, tumor necrosis factor; IL-2R, interleukin-2 receptor. 


\section{Author contributions}

GP and MT-conceptualization; MT and GPmethodology; MT and GP-formal analysis; MTinvestigation; MT-resources; GP and LB-data curation; $\mathrm{MT}$ and $\mathrm{GP}$ - writing original draft preparation; LB, IP and $\mathrm{JP}$-writing review and editing; GP and JPvisualization; GP - supervision; GP and LB - project administration; LB - funding acquisition. All authors have read and agreed to the published version of the manuscript.

\section{Ethics approval and consent to participate}

Not applicable.

\section{Acknowledgment}

Thanks to all the peer reviewers for their opinions and suggestions.

\section{Funding}

This work was supported by the Ministry of Health of the Czech Republic project for conceptual development in research organizations, ref. no. 65269705 (University Hospital Brno, Brno, Czech Republic).

\section{Conflict of interest}

The authors declare no conflict of interest.

\section{References}

[1] Dhakal BP, Sweitzer NK, Indik JH, Acharya D, William P. SARS-CoV-2 Infection and Cardiovascular Disease: COVID19 Heart. Heart, Lung and Circulation. 2020; 29: 973-987.

[2] World Health Organization. WHO Coronavirus (COVID-19) Dashboard. 2021. Available at: https://covid19.who.int/ (Accessed: 24 September 2021).

[3] Wilder-Smith A, Chiew CJ, Lee VJ. Can we contain the COVID19 outbreak with the same measures as for SARS? The Lancet Infectious Diseases. 2020; 20: e102-e107.

[4] Cao Y, Liu X, Xiong L, Cai K. Imaging and clinical features of patients with 2019 novel coronavirus SARS-CoV-2: a systematic review and meta-analysis. Journal of Medical Virology. 2020; 92: 1449-1459.

[5] Wu Z, McGoogan JM. Characteristics of and Important Lessons from the Coronavirus Disease 2019 (COVID-19) Outbreak in China: summary of a report of 72314 cases from the Chinese Center for Disease Control and Prevention. Journal of the American Medical Association. 2020; 323: 1239.

[6] Wang D, Hu B, Hu C, Zhu F, Liu X, Zhang J, et al. Clinical Characteristics of 138 Hospitalized Patients with 2019 Novel Coronavirus-Infected Pneumonia in Wuhan, China. Journal of the American Medical Association. 2020; 323: 1061.

[7] Inciardi RM, Lupi L, Zaccone G, Italia L, Raffo M, Tomasoni D, et al. Cardiac Involvement in a Patient With Coronavirus Disease 2019 (COVID-19). JAMA Cardiology. 2020; 5: 819-824.

[8] Madjid M, Safavi-Naeini P, Solomon SD, Vardeny O. Potential Effects of Coronaviruses on the Cardiovascular System: A Review. JAMA Cardiology. 2020; 5: 831.

[9] Nuzzi V, Merlo M, Specchia C, Lombardi CM, Carubelli V, Iorio $\mathrm{A}$, et al. The prognostic value of serial troponin measurements in patients admitted for COVID-19. ESC Heart Failure. 2021; 8: 3504-3511.
[10] Huang C, Wang Y, Li X, Ren L, Zhao J, Hu Y, et al. Clinical features of patients infected with 2019 novel coronavirus in Wuhan, China. The Lancet. 2020; 395: 497-506.

[11] Song P, Li W, Xie J, Hou Y, You C. Cytokine storm induced by SARS-CoV-2. Clinica Chimica Acta. 2020; 509: 280-287.

[12] Guan H, Liu J, Ding J, Liu W, Feng Y, Bao Y, et al. Arrhythmias in patients with coronavirus disease 2019 (COVID-19) in Wuhan, China: Incidences and implications. Journal of Electrocardiology. 2021; 65: 96-101.

[13] Hu B, Guo H, Zhou P, Shi Z. Characteristics of SARS-CoV-2 and COVID-19. Nature Reviews Microbiology. 2021; 19: 141-154.

[14] Du Y, Tu L, Zhu P, Mu M, Wang R, Yang P, et al. Clinical Features of 85 Fatal Cases of COVID-19 from Wuhan. a Retrospective Observational Study. American Journal of Respiratory and Critical Care Medicine. 2020; 201: 1372-1379.

[15] Wu Z, McGoogan JM. Characteristics of and Important Lessons from the Coronavirus Disease 2019 (COVID-19) Outbreak in China: Summary of a Report of 72314 Cases From the Chinese Center for Disease Control and Prevention. Journal of the American Medical Association. 2020; 323: 1239.

[16] Banks E, Welsh J, Joshy G, Martin M, Paige E, Korda RJ. Comparison of cardiovascular disease risk factors, assessment and management in men and women, including consideration of absolute risk: a nationally representative cross-sectional study. BMJ Open. 2020; 10: e038761.

[17] Roth GA, Johnson C, Abajobir A, Abd-Allah F, Abera SF, Abyu $\mathrm{G}$, et al. Global, Regional, and National Burden of Cardiovascular Diseases for 10 Causes, 1990 to 2015. Journal of the American College of Cardiology. 2017; 70: 1-25.

[18] Ruan Y, Guo Y, Zheng Y, Huang Z, Sun S, Kowal P, et al. Cardiovascular disease (CVD) and associated risk factors among older adults in six low-and middle-income countries: results from SAGE Wave 1. BMC Public Health. 2018; 18: 778.

[19] Burns PB, Rohrich RJ, Chung KC. The levels of evidence and their role in evidence-based medicine. Plastic and Reconstructive Surgery. 2011; 128: 305-310.

[20] Vlachakis PK, Tentolouris A, Tousoulis D, Tentolouris N. Current data on the cardiovascular effects of COVID-19. Hellenic Journal of Cardiology. 2020; 61: 46-48.

[21] Amirfakhryan H, safari F. Outbreak of SARS-CoV2: Pathogenesis of infection and cardiovascular involvement. Hellenic Journal of Cardiology. 2021; 62: 13-23.

[22] Michas G, Karvelas G, Trikas A. Cardiovascular disease in Greece; the latest evidence on risk factors. Hellenic Journal of Cardiology. 2019; 60: 271-275.

[23] Azevedo RB, Botelho BG, Hollanda JVGD, Ferreira LVL, Junqueira de Andrade LZ, Oei SSML, et al. Covid-19 and the cardiovascular system: a comprehensive review. Journal of Human Hypertension. 2021; 35: 4-11.

[24] Zhou P, Yang XL, Wang XG, Hu B, Zhang L, Zhang W, et al. A pneumonia outbreak associated with a new coronavirus of probable bat origin. Nature. 2020; 579: 270-273.

[25] Zhou Y, Chi J, Lv W, Wang Y. Obesity and diabetes as highrisk factors for severe coronavirus disease 2019 (Covid-19). Diabetes/Metabolism Research and Reviews. 2021; 37: e3377.

[26] Bae S, Kim SR, Kim M, Shim WJ, Park S. Impact of cardiovascular disease and risk factors on fatal outcomes in patients with COVID-19 according to age: a systematic review and metaanalysis. Heart. 2021; 107: 373-380.

[27] Guo W, Li M, Dong Y, Zhou H, Zhang Z, Tian C, et al. Diabetes is a risk factor for the progression and prognosis of COVID-19. Diabetes/Metabolism Research and Reviews. 2020: e3319.

[28] Silverio A, Di Maio M, Citro R, Esposito L, Iuliano G, Bellino $\mathrm{M}$, et al. Cardiovascular risk factors and mortality in hospitalized patients with COVID-19: systematic review and meta-analysis of 45 studies and 18,300 patients. BMC Cardiovascular Disorders. 2021; 21: 23 . 
[29] Shang J, Wang Q, Zhang H, Wang X, Wan J, Yan Y, et al. The Relationship between Diabetes Mellitus and COVID-19 Prognosis: a Retrospective Cohort Study in Wuhan, China. The American Journal of Medicine. 2021; 134: e6-e14.

[30] Hsu CM, Weiner DE, Aweh G, Miskulin DC, Manley HJ, Stewart C, et al. COVID-19 among us Dialysis Patients: Risk Factors and Outcomes from a National Dialysis Provider. American Journal of Kidney Diseases. 2021; 77: 748-756.e1.

[31] Kuroda S, Matsumoto S, Sano T, Kitai T, Yonetsu T, Kohsaka S, et al. External validation of the 4C Mortality Score for patients with COVID-19 and pre-existing cardiovascular diseases/risk factors. BMJ Open. 2021; 11: e052708.

[32] Li J, You Z, Wang Q, Zhou Z, Qiu Y, Luo R, et al. The epidemic of 2019-novel-coronavirus (2019-nCoV) pneumonia and insights for emerging infectious diseases in the future. Microbes and Infection. 2020; 22: 80-85.

[33] de Almeida-Pititto B, Dualib PM, Zajdenverg L, Dantas JR, de Souza FD, Rodacki M, et al. Severity and mortality of COVID 19 in patients with diabetes, hypertension and cardiovascular disease: a meta-analysis. Diabetology \& Metabolic Syndrome. 2020; $12: 75$.

[34] Ghany R, Palacio A, Chen G, Dawkins E, McCarter D, Forbes $\mathrm{E}$, et al. Prior cardiovascular risk and screening echocardiograms predict hospitalization and severity of coronavirus infection among elderly medicare patients. American Journal of Preventive Cardiology. 2020; 3: 100090.

[35] Frydrych LM, Bian G, O’Lone DE, Ward PA, Delano MJ. Obesity and type 2 diabetes mellitus drive immune dysfunction, infection development, and sepsis mortality. Journal of Leukocyte Biology. 2018; 104: 525-534.

[36] Kalligeros M, Shehadeh F, Mylona EK, Benitez G, Beckwith CG, Chan PA, et al. Association of Obesity with Disease Severity among Patients with Coronavirus Disease 2019. Obesity. 2020; 28: 1200-1204.

[37] Chu Y, Yang J, Shi J, Zhang P, Wang X. Obesity is associated with increased severity of disease in COVID-19 pneumonia: a systematic review and meta-analysis. European Journal of Medical Research. 2020; 25: 64.

[38] Cava E, Neri B, Carbonelli MG, Riso S, Carbone S. Obesity pandemic during COVID-19 outbreak: Narrative review and future considerations. Clinical Nutrition. 2021; 40: 1637-1643.

[39] Yang J, Tian C, Chen Y, Zhu C, Chi H, Li J. Obesity aggravates COVID-19: an updated systematic review and meta-analysis. Journal of Medical Virology. 2021; 93: 2662-2674.

[40] Lippi G, Henry BM. Active smoking is not associated with severity of coronavirus disease 2019 (COVID-19). European Journal of Internal Medicine. 2020; 75: 107-108.

[41] Landoni G, Moro M, Belletti A, Rovere-Querini P, Veronesi G, Ruggeri A, et al. Recent exposure to smoking and COVID-19. Critical Care and Resuscitation. 2020; 22: 253-256.

[42] Patanavanich R, Glantz SA. Smoking is Associated with COVID-19 Progression: a Meta-analysis. Nicotine \& Tobacco Research. 2020; 22: 1653-1656.

[43] Reddy RK, Charles WN, Sklavounos A, Dutt A, Seed PT, Khajuria A. The effect of smoking on COVID-19 severity: A systematic review and meta-analysis. Journal of Medical Virology. 2021; 93: 1045-1056.

[44] Pepera G, Panagiota Z. Comparison of heart rate response and heart rate recovery after step test among smoker and non-smoker athletes. African Health Sciences. 2021; 21: 105-111.

[45] Xia G, Fan D, Ma C, He Y, Wang M, Zhu Y, et al. HyperInflammatory Response Involves in Cardiac Injury among Patients with Coronavirus Disease 2019. The American Journal of the Medical Sciences. 2021; 361: 718-724.

[46] Tay MZ, Poh CM, Rénia L, MacAry PA, Ng LFP. The trinity of COVID-19: immunity, inflammation and intervention. Nature
Reviews Immunology. 2020; 20: 363-374.

[47] Costantini S, Sharma A, Colonna G. The Value of the Cytokinome Profile, Inflammatory Diseases - A Modern Perspective. Dr. Amit Nagal (ed.) 2011. Available at: http://www.intechopen.com/books/inflammatory-diseases-a -modern-perspective/the-value-of-the-cytokinome-profile (Accessed: 20 November 2021).

[48] Busana M, Gasperetti A, Giosa L, Forleo GB, Schiavone M, Mitacchione G, et al. Prevalence and outcome of silent hypoxemia in COVID-19. Minerva Anestesiologica. 2021; 87: 325-333.

[49] Bickler PE, Feiner JR, Lipnick MS, McKleroy W. "Silent" Presentation of Hypoxemia and Cardiorespiratory Compensation in COVID-19. Anesthesiology. 2021; 134: 262-269.

[50] Simonson TS, Baker TL, Banzett RB, Bishop T, Dempsey JA, Feldman JL, et al. Silent hypoxaemia in COVID-19 patients. Journal of Physiology. 2021; 599: 1057-1065.

[51] Sorokin AV, Karathanasis SK, Yang Z, Freeman L, Kotani K, Remaley AT. COVID-19-Associated dyslipidemia: Implications for mechanism of impaired resolution and novel therapeutic approaches. The FASEB Journal. 2020; 34: 9843-9853.

[52] Izcovich A, Ragusa MA, Tortosa F, Lavena Marzio MA, Agnoletti C, Bengolea A, et al. Prognostic factors for severity and mortality in patients infected with COVID-19: A systematic review. PLoS ONE. 2020; 15: e0241955.

[53] Mitacchione G, Schiavone M, Curnis A, Arca M, Antinori S, Gasperetti A, et al. Impact of prior statin use on clinical outcomes in COVID-19 patients: data from tertiary referral hospitals during COVID-19 pandemic in Italy. Journal of Clinical Lipidology. 2021; 15: 68-78.

[54] COVIDNet. Centers for Disease Control and Prevention. Laboratory-Confirmed COVID-19-Associated Hospitalizations. 2021. Available at: https://gis.cdc.gov/grasp/COVIDNet /COVID19_5.html\#medicalConditionsColumnDiv (Accessed: 17 July 2021).

[55] Pareek M, Singh A, Vadlamani L, Eder M, Pacor J, Park J, et al. Relation of Cardiovascular Risk Factors to Mortality and Cardiovascular Events in Hospitalized Patients With Coronavirus Disease 2019 (from the Yale COVID-19 Cardiovascular Registry). American Journal of Cardiology. 2021; 146: 99-106.

[56] Lazaridis C, Vlachogiannis NI, Bakogiannis C, Spyridopoulos I, Stamatelopoulos K, Kanakakis I, et al. Involvement of cardiovascular system as the critical point in coronavirus disease 2019 (COVID-19) prognosis and recovery. Hellenic Journal of Cardiology. 2020; 61: 381-395.

[57] Haanen C, Vermes I. Apoptosis and inflammation. Mediators of Inflammation. 1995; 4: 5-15.

[58] Yang Y, Jiang G, Zhang P, Fan J. Programmed cell death and its role in inflammation. Military Medical Research. 2015; 2: 12.

[59] Nailwal H, Chan FK. Necroptosis in anti-viral inflammation. Cell Death \& Differentiation. 2019; 26: 4-13.

[60] Yu B, Yu L, Klionsky DJ. Nutrition acquisition by human immunity, transient overnutrition and the cytokine storm in severe cases of COVID-19. Medical Hypotheses. 2021; 155: 110668.

[61] Grundy SM. Overnutrition, ectopic lipid and the metabolic syndrome. Journal of Investigative Medicine. 2016; 64: 1082-1086.

[62] Virtue S, Vidal-Puig A. Adipose tissue expandability, lipotoxicity and the Metabolic Syndrome - an allostatic perspective. Biochimica Et Biophysica Acta (BBA) - Molecular and Cell Biology of Lipids. 2010; 1801: 338-349.

[63] van Niekerk G, du Toit A, Loos B, Engelbrecht A. Nutrient excess and autophagic deficiency: explaining metabolic diseases in obesity. Metabolism: Clinical and Experimental. 2018; 82: $14-21$.

[64] Summers SA. Ceramides in insulin resistance and lipotoxicity. Progress in Lipid Research. 2006; 45: 42-72.

[65] van Herpen NA, Schrauwen-Hinderling VB. Lipid accumulation 
in non-adipose tissue and lipotoxicity. Physiology \& Behavior. 2008; 94: 231-241.

[66] Mitacchione G, Schiavone M, Gasperetti A, Forleo GB. Ventricular tachycardia storm management in a COVID-19 patient: a case report. European Heart Journal - Case Reports. 2020; 4: $1-6$.

[67] Yasmin Kusumawardhani N, Huang I, Martanto E, Sihite TA, Nugraha ES, Prodjosoewojo S, et al. Lethal Arrhythmia (Torsade de Pointes) in COVID-19: an Event Synergistically Induced by Viral Associated Cardiac Injury, Hyperinflammatory Response, and Treatment Drug? Clinical Medicine Insights: Case Reports. 2020; 13: 117954762097239.

[68] Altamimi H, Abid AR, Othman F, Patel A. Cardiovascular Manifestations of COVID-19. Heart Views. 2020; 21: 171-186.

[69] Lai C, Shih T, Ko W, Tang H, Hsueh P. Severe acute respiratory syndrome coronavirus 2 (SARS-CoV-2) and coronavirus disease-2019 (COVID-19): the epidemic and the challenges. International Journal of Antimicrobial Agents. 2020; 55: 105924.

[70] Stefanikis M, Batalik L, Papathanasiou J, Dipla L, Antoniou V, Pepera G. Exercise-based cardiac rehabilitation programs in the era of COVID19: A critical review. Reviews in Cardiovascular Medicine. 2021; 22: 1143-1155.

[71] Barker-Davies RM, O'Sullivan O, Senaratne KPP, Baker P, Cranley M, Dharm-Datta S, et al. The Stanford Hall consensus statement for post-COVID-19 rehabilitation. British Journal of Sports Medicine. 2020; 54: 949-959.

[72] Calabrese M, Garofano M, Palumbo R, Di Pietro P, Izzo C, Damato A, et al. Exercise Training and Cardiac Rehabilitation in COVID-19 Patients with Cardiovascular Complications: State of Art. Life. 2021; 11: 259.

[73] Batalik L, Konecny V, Dosbaba F, Vlazna D, Brat K.Cardiac Rehabilitation Based on the Walking Test and Telerehabilitation Improved Cardiorespiratory Fitness in People Diagnosed with Coronary Heart Disease during the COVID-19 Pandemic. International Journal of Environmental Research and Public Health. 2021; 18: 2241.

[74] Batalik L, Dosbaba F, Hartman M, Konecny V, Batalikova K, Spinar J. Long-term exercise effects after cardiac telerehabilitation in patients with coronary artery disease: 1-year follow-up results of the randomized study. European Journal of Physical and Rehabilitation Medicine. 2021; 57: 807-814.

[75] Sandercock GRH, Cardoso F, Almodhy M, Pepera G. Cardiorespiratory fitness changes in patients receiving comprehensive outpatient cardiac rehabilitation in the UK: a multicentre study. Heart. 2013; 99: 785-790.

[76] Pepera G, Cardoso F, Taylor MJD, Peristeropoulos A, Sandercock GRH. Predictors of shuttle walking test performance in patients with cardiovascular disease. Physiotherapy. 2013; 99: 317-322.

[77] Cardoso FMF, Almodhy M, Pepera G, Stasinopoulos DM, Sandercock GRH. Reference values for the incremental shuttle walk test in patients with cardiovascular disease entering exercise-based cardiac rehabilitation. Journal of Sports Sciences. 2017; 35: 1-6.

[78] Pepera G, Bromley P, Sandercock G. A pilot study to investigate the safety of exercise training and testing in cardiac rehabilitation patients. British Journal of Cardiology. 2013; 20: 78. 